\title{
LSDCat: Detection and cataloguing of emission-line sources in integral-field spectroscopy datacubes (Corrigendum)
}

\author{
Edmund Christian Herenz ${ }^{1}$ and Lutz Wisotzki ${ }^{2}$ \\ 1 European Southern Observatory, Av. Alonso de Córdova 3107, 7630355 Vitacura, Santiago, Chile \\ e-mail: eherenz@eso.org \\ ${ }^{2}$ Leibniz-Institut für Astrophysik Potsdam (AIP), An der Sternware 16, 14482 Potsdam, Germany \\ A\&A 602, A111 (2017), https://doi .org/10.1051/0004-6361/201629507
}

Key words. methods: data analysis - techniques: imaging spectroscopy - errata, addenda

We correct an error in Eq. (34) of Herenz \& Wisotzki (2017), where we provided an estimate for the signal-to-noise ratio for an emission line that matches the 3D Gaussian filter template of LSDCat. The correct equation reads

$S / N_{x^{\prime}, y^{\prime}, z^{\prime}} \approx \frac{1}{\sqrt{8 \pi^{3 / 2} \sigma_{z}} \sigma_{\mathrm{G}}} \times \frac{F_{\text {line }}}{\bar{\sigma} \Delta \lambda}$,

that is, $\sigma_{G}$ is not under the square root as presented in the paper. All previous steps that lead to this equation, that is, Eqs. (29)(33), were correct.

As a consequence of this correction, the numerical value of the limiting flux in the MUSE HDF-S data at $7000 \AA$, given in Eq. (35) of our paper, was also incorrect. Inserting the given values $\left(\sigma_{\mathrm{G}}=1.84 \mathrm{px}, \sigma_{z}=1.46, \bar{\sigma}=1.42 \times 10^{-20} \mathrm{erg} \mathrm{s}^{-1} \mathrm{~cm}^{-2} \AA^{-1}\right.$, $\Delta \lambda=1.2 \AA$, and $S / N_{\mathrm{det}} \approx 8$ ) into Eq. (1) delivers

$\log F_{\text {line }}\left[\mathrm{erg} \mathrm{s}^{-1} \mathrm{~cm}^{-2}\right] \approx-17.69$.

Therefore Fig. 3, where the theoretical limiting flux was compared with the results of a source insertion and recovery experiment, also needs to be corrected. The corrected version of Fig. 3 is shown here in Fig. 1. The match between the experiment and theoretical expectation in Fig. 1 is closer in comparison to what was originally presented, so parts of the discussion in the final paragraph of Sect. 3.2 are thus obsolete.

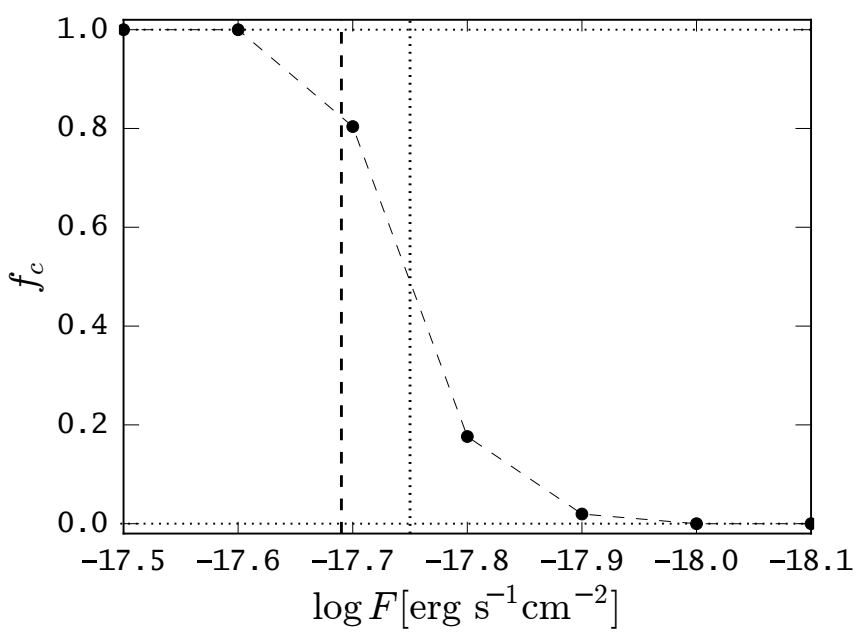

Fig. 1. Completeness curve $f_{\mathrm{C}}\left(\log F\left[\mathrm{erg} \mathrm{s}^{-1} \mathrm{~cm}^{-2} \AA^{-1}\right]\right)$ from a fake source insertion and recovery experiment on the MUSE HDF-S datacube at $5000 \AA$. The dotted vertical line shows the $50 \%$ completeness limit at $\log F\left[\mathrm{erg} \mathrm{s}^{-1} \mathrm{~cm}^{-2}\right]=-17.75$ and the dashed vertical line shows the analytically approximated minimum line flux given in Eq. (2) as $\log F_{\text {line }}\left[\mathrm{erg} \mathrm{s}^{-1} \mathrm{~cm}^{-2}\right] \approx-17.69$ at which LSDCat is expected to detect emission line sources in this experiment.

\section{References}

Herenz, E. C., \& Wisotzki, L. 2017, A\&A, 602, A111 